\title{
SURGICAL MANAGEMENT OF SEVERE SPONTANEOUS HEMORRHAGE OF THE ABDOMINAL WALL COMPLICATING ACENOCOUMAROL TREATMENT
}

\author{
Orestis Ioannidis $^{1}$, George Paraskevas ${ }^{2}$, Anastasios Kotronis ${ }^{1}$, Stavros Chatzopoulos ${ }^{1}$, Athina Konstantara ${ }^{1}$, \\ Nikolaos Papadimitriou ${ }^{1}$, Apostolos Makrantonakis ${ }^{1}$, Emmanouil Kakoutis ${ }^{1}$
}

First Surgical Department, General Regional Hospital 'George Papanikolaou'. Thessaloniki, Greece'; Department of Anatomy, Medical School, Aristotle University of Thessaloniki, Greece²

Summary: Acenocoumarol is a vitamin K antagonist that is used for the treatment of acquired and congenital, both arterial and venous, thrombotic diseases. Its use is complicated by the narrow therapeutic range. Bleeding following oral anticoagulation, despite rare, remains the major complication. Most cases of hemorrhagic episodes usually require short hospitalization and transfusion, while surgical drainage of the hematoma is not recommended. However, in cases that conservative treatment isn't successful, surgical intervention remains an option. We present a case of severe spontaneous bleeding of the rectus abdominis muscle which was successfully managed surgically.

Key words: Oral anticoagulants; Bleeding; Hematoma; Surgery; Abdominis rectus muscle

\section{Introduction}

Oral anticoagulants are vitamin $\mathrm{K}$ antagonists and include coumarins (warfarin and acenocoumarol) and indandione derivatives (fenindione) $(5,6)$. Coumarins are used worldwide and they are the treatment of choice for primary and secondary prevention for an increasing variety of acquired and congenital thrombotic diseases, both arterial and venous $(2,13,14)$. Coumarins produce their effect by inhibiting vitamin $\mathrm{K}$ epoxide reductase and quinone reductase (6), and depressing the function of thrombin, prothrombin, and factors VII, IX and $X$, and proteins $C$ and $S(5,6,15)$. The clinical use of coumarins is complicated by the large pharmacokinetic and pharmacodynamic interindividual variability and narrow therapeutic range $(7,11,13)$. While bleeding following oral anticoagulation therapy is rare (5), it still remains the more serious complication ranging from minor bleeding to severe, spontaneous hemorrhages (14, 15). We present a case of severe spontaneous bleeding of the rectus abdominis muscle which was successfully managed surgically.

\section{Case report}

A 70 year old female presented to the emergency department of our hospital with sudden onset of acute abdominal pain and rigor. The patient was hemodynamically unstable with an arterial pressure of $97 / 44 \mathrm{mmHg}$ and a heart rate of 128 beats per minute. Oxygen saturation was 97\%. Past medical history revealed arterial hypertension, diabetes mellitus, hyperlipidemia, hyperuricemia, coronary disease, aortic valve deficiency and chronic atrial fiblilation treated for the last 3 years with acenocoumarol 2 and $3 \mathrm{mg}$ every other day. There was no history of recent abdominal trauma, no changes in patient's medication and diet and no recent concurrent disease.

Physical examination revealed an anxious female patient with a palpable mass of the right abdominal wall, especially in the right side mostly in the sheath of the abdominis rectus muscle. Laboratory examination showed anemia (hematocrit $24.7 \%$ and hemoglobin $8.2 \mathrm{~g} / \mathrm{dl}$ ) with normal white blood cells count $(9.1 \mathrm{~K} / \mathrm{mL})$ and platelets count $(276 \mathrm{~K} / \mathrm{mL})$, hyperamylasemia (1104 IU/L), slightly increased SGOT (63 IU/L) and SGPT (48 IU/L) and CPK (427 IU/L) and acute renal failure (urea $285 \mathrm{mg} / \mathrm{dL}$ and creatinine $3.6 \mathrm{mg} / \mathrm{dL}$ ). Also, abnormalities in hemostasis were present with a PT 30" (with the control being 11"), a PTT 48" (with the control being 28") and an INR 3.01.

Abdominal ultrasonography revealed a sizable abdominal mass measuring about $12 \times 8 \mathrm{~cm}$ which could be attributed to a hematoma or tumor. The abdominal CT demonstrated a large hematoma in the sheath of the abdominis rectus and the right oblique abdominal muscles (Figure 1).

The patient initially was managed with conservative treatment, including discontinuation of acenocoumarol, pause of oral feeding and administration of parenteral fluids, 3 units of whole blood, 4 units of fresh frozen plasma and $20 \mathrm{mg}$ of vitamin K intravenously. Despite the supportive 


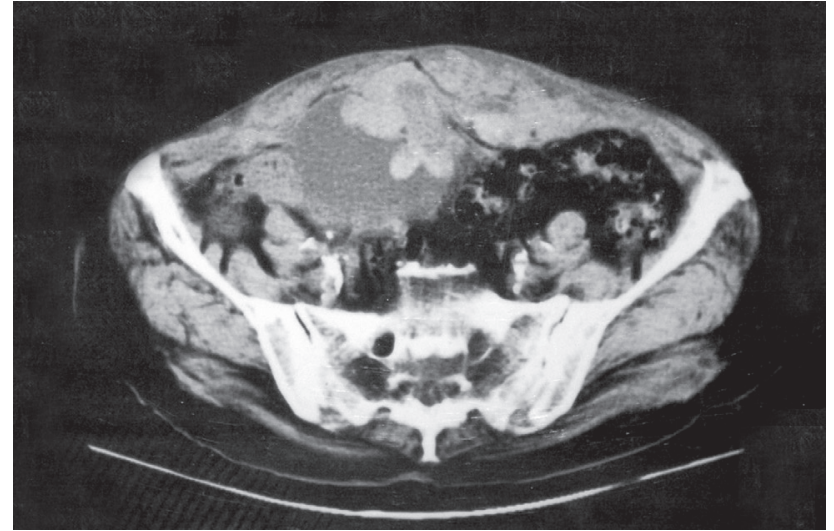

Fig. 1: The abdominal CT scan demonstrated a sizable hematoma of the sheath of the right rectus abdominis muscle

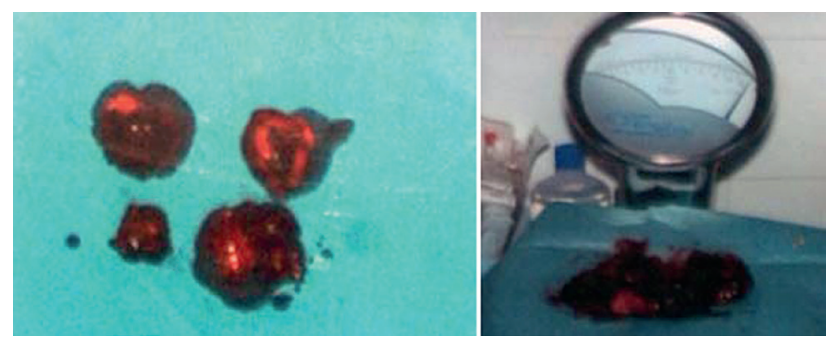

Fig. 2: The removed blood clots weighed about 200 gr.

treatment the patient didn't show any clinical improvement. Specifically, the patient remained hemodynamically unstable, experienced tachypnea and dyspnea, the oxygen saturation dropped to $86 \%$ in the air and was elevated to $93 \%$ with 4 liters of oxygen per minute. Also the patient presented oliguria. The INR had been partially corrected after initial treatment (INR 1.7) but the anemia persisted (hematocrit $25.4 \%$ and hemoglobin $8.7 \mathrm{~g} / \mathrm{dl}$ ) despite transfusion. Surgical intervention was decided on the basis that despite conservative treatment the patient's clinical condition wasn't improving and anemia persisted indicating that there was continuing active bleeding. So, taking into account that the hemostatic mechanism was partially corrected and the anticoagulation therapy reversed, 18 hours after submission the surgical intervention was performed. Intraoperatively, a large hematoma originating from the rectus abdominis and the oblique abdominal muscles was observed. The abdominal muscles were oozing, presented capillary hemorrhage and were fragile. All clots were removed and compression, electrocauterization and ligation were used for hemostasis. Also haemostatic powder was used and a drainage was placed. The evacuated blood clots weighed approximately 200 gr (Figure 2).

The patient improved posroperatively. The laboratory findings 24 hours postoperatively were hematocrit $30.2 \%$ and hemoglobin $10.4 \mathrm{~g} / \mathrm{dl}$, amylase $362 \mathrm{IU} / \mathrm{L}$, SGOT $45 \mathrm{IU} / \mathrm{L}$,
SGPT 24 IU/L, CPK $328 \mathrm{IU} / \mathrm{L}$, urea $69 \mathrm{mg} / \mathrm{dL}$, creatinine $1.2 \mathrm{mg} / \mathrm{dL}$, PT 13" (with the control being 12"), PTT 34 (with the control being 32") and INR 1.09. The patient was discharged on the 12th postoperative day.

\section{Discussion}

Coumarins are among the most commonly prescribed drugs (14) and the most common indications include atrial fibrillation, prevention and treatment of venous thromboembolism, heart valve replacement, valvular heart disease and ischemic and nonischemic heart disease $(9,15)$. However, bleeding following oral anticoagulation, despite rare, remains the major complication $(5,15)$. The reported frequency of major hemorrhage secondary to acenocoumarol treatment is 2.39 episodes per 100 patient-years and for fatal episodes secondary to acenocoumarol treatment is 0.1 episodes per 100 patient-years (2). The most common sites of hemorrhage are genitourinary, gastrointestinal, intracranial, oropharyngeal and cutaneous (5, 15), while regarding acenocoumarol the usual locations are gastroiontestinal, epistaxis and central nervous system (2). Spontaneous bleeding of the rectus abdominis muscle and hematoma formation of the abdominal wall is rare. The most important risk factors for bleeding in patients receiving acenocoumarol are the deviation of the INR from the target range and the intensity of anticoagulation, with each unit increase in the INR raising the risk of bleeding by 3.5 times $(2,3,15)$. Other potential causative factors include errors in anticoagulant administration, comorbid conditions, age (over 75 years) dietary habits and interaction with other medications $(2,5,15)$. Furthermore, in the last few years with the advance of pharmacogenomics the influence of genetic factors in oral anticoagulation has become evident. Polymorphisms of CYP2C9, VKORC1, $\mathrm{ABCB} 1$ and CYP4F2 genes affect the dose requirements $(10,11,14)$.

A highly sensitive and specific sign of a coagulopathic hemorrhage is the CT finding of the hematocrit effect, which is observed with increased frequency in patients receiving oral anticoagulants (4). The hematocrit effect is the linear separation of cellular and liquid components of blood and settling of the cellular elements in the dependent position of the hematoma (4).

Most cases of major hemorrhagic episodes don't have a long-term sequale but usually require short hospitalization and transfusion (3). The treatment of bleeding is initially supportive and medical and immediate correction of the coagulation disturbance is mandatory $(5,15)$. The oral anticoagulation is necessary to be stopped and administration of fresh frozen plasma or prothrombin complex concentrate along with intravenous vitamin $\mathrm{K}$ is recommended. Also, the administration of recombinant factor VIIa seems to be effective (7). However, increased attention is needed in order to balance the risk of bleeding against the potential risk of thromboembolism (15). Surgical drainage of the hema- 
toma is not recommended as the natural course of bleeding is the spontaneous disappearance of the hematoma $(5,15)$. Although in cases that the patient doesn't respond to supportive measures surgical intervention can be helpful and shouldn't be delayed (12), as in the present case where despite the transfusion of fresh frozen plasma and whole blood and intravenous vitamin $\mathrm{K}$ administration the patient's clinical condition wasn't improving so a surgical intervention was decided which succeeded to drain the hematoma and control the bleeding. The most fatal major bleeding episodes are intracelebral $(2,3)$.

\section{Conclusions}

Close and regular INR monitoring and adhering as closely as possible to the recommended therapeutic INR ranges is necessary in order to identify patients at risk of bleeding and to decrease the frequency of overanticoagulation and related hemorrhage $(1,8)$. However, even in patients with an INR in the upper limit of the therapeutic range severe spontaneous bleeding may occur. In the present case, the INR value is high regarding the surgeons perspective as there will be excessive bleeding in the surgical field but it isn't considered extremely high. On the other hand the INR value is very close to the desired therapeutic range, practically its upper limit, so it cannot be considered high from this point of view. However, despite that fact the patient presented with extensive spontaneous bleeding. Most cases of hemorrhagic episodes usually require short hospitalization and transfusion, while surgical drainage of the hematoma is not recommended. However, in cases that conservative treatment isn't successful, surgical intervention remains an option and shouldn't be delayed. Reversal of anticoagulation therapy is required prior to surgery.

\section{References}

1. Casais P, Luceros AS, Meschengieser S, Bermejo E, Lazzari MA. INR variability in anticoagulation with acenocoumarol: is it useful for identifying patients at risk of bleeding and thrombosis? Haematologica 1999; 84: 753-4.

2. Casais P, Luceros AS, Meschengieser S, Fondevila C, Santarelli MT, Lazzari MA. Bleeding risk factors in chronic oral anticoagulation with acenocoumarol. Am J Hematol 2000; 63: 192-6.

3. Eckman MH, Levine HJ, Pauker SG. Effect of laboratory variation in the prothrombin-time ratio on the results of oral anticoagulant therapy. N Engl J Med 1993; 329: 696-702.

4. Federle MP, Pan KT, Pealer KM. CT criteria for differentiating abdominal hemorrhage: anticoagulation or aortic aneurysm rupture? AJR Am J Roentgenol 2007; 188: $1324-30$

5. González-García R, Schoendorff G, Muñoz-Guerra MF, Rodríguez-Campo FJ, Naval-Gías L, Sastre-Pérez J. Upper airway obstruction by sublingual hematoma: a complication of anticoagulation therapy with acenocoumarol. Am J Otolaryngol 2006; 27: 129-32.

6. Hirsh J, Fuster V. Guide to anticoagulant therapy. Part 2: Oral anticoagulants. American Heart Association. Circulation 1994; 89: 1469-80.

7. Ingerslev J, Vanek T, Culic S. Use of recombinant factor VIIa for emergency reversal of anticoagulation. J Postgrad Med 2007; 53: 17-22.

8. Koo S, Kucher N, Nguyen PL, Fanikos J, Marks PW, Goldhaber SZ. The effect of excessive anticoagulation on mortality and morbidity in hospitalized patients with anticoagulant-related major hemorrhage. Arch Intern Med 2004; 164: 1557-60.

9. Levi M, Hovingh GK, Cannegieter SC, Vermeulen M, Büller HR, Rosendaal FR. Bleeding in patients receiving vitamin $\mathrm{K}$ antagonists who would have been excluded from trials on which the indication for anticoagulation was based. Blood 2008; 111: 4471-6.

10. Pérez-Andreu V, Roldán V, Antón AI, et al. Pharmacogenetic relevance of CYP4F2 V433M polymorphism on acenocoumarol therapy. Blood 2009; 113: 4977-9.

11. Saraeva RB, Paskaleva ID, Doncheva E, Eap CB, Ganev VS. Pharmacogenetics of acenocoumarol: CYP2C9, CYP2C19, CYP1A2, CYP3A4, CYP3A5 and ABCB1 gene polymorphisms and dose requirements. J Clin Pharm Ther 2007; 32: 641-9.

12. Topgül K, Uzun O, Anadol AZ, Gök A. Surgical management of enoxaparin- and/ or warfarin-induced massive retroperitoneal bleeding: report of a case and review of the literature. South Med J 2005; 98: 104-6.

13. Vayá A, Martínez M, Fernández A, et al. The effect of acenocoumarol on hemorheological parameters. Clin Hemorheol Microcirc 2001; 24: 111-5.

14. Verde Z, Ruiz JR, Santiago C, Valle B, et al. A novel, single algorithm approach to predict acenocoumarol dose based on CYP2C9 and VKORC1 allele variants. PLoS One 2010; 5: e11210.

15. Wey PF, Petitjeans F, Lions C, Ould-Ahmed M, Escarment J. Laryngeal dyspnea in relation to an interaction between acenocoumarol and topical econazole lotion. Am J Geriatr Pharmacother 2008; 6: 173-7.

Received: $23 / 06 / 2011$

Accepted in revised form: 20/01/2012

\section{Corresponding author:}

Orestis Ioannidis, Alexandrou Mihailidi 13, 54640 Thessaloniki, Greece; e-mail: telonakos@hotmail.com 